\title{
Postoperative Radiographic Early-Onset Adjacent Segment Degeneration after Single-Level L4-L5 Posterior Lumbar Interbody Fusion in Patients without Preoperative Severe Sagittal Spinal Imbalance
}

Yuji Matsuoka, Kenji Endo, Hidekazu Suzuki, Yasunobu Sawaji, Hirosuke Nishimura, Taichiro Takamatsu, Osamu Kojima, Kazuma Murata, Takeshi Seki, Shinji Horie, Takamitsu Konishi, Kengo Yamamoto

Department of Orthopedic Surgery, Tokyo Medical University, Tokyo, Japan

Study Design: Retrospective study.

Purpose: To investigate the relationship between preoperative total spinal sagittal alignment and the early onset of adjacent segment degeneration (ASD) after single-level posterior lumbar interbody fusion (PLIF) in patients with normal sagittal spinal alignment. Overview of Literature: Postoperative early-onset ASD is one of the complications after L4-L5 PLIF, a common surgical procedure for lumbar degenerative disease in patents without severe sagittal imbalance. A better understanding of the preoperative characteristics of total spinal sagittal alignment associated with early-onset ASD could help prevent the condition.

Methods: The study included 70 consecutive patients diagnosed with lumbar degenerative disease who underwent single-level L4L5 PLIF between 2011 and 2015. They were divided into two groups based on the radiographic progression of L3-L4 degeneration after 1-year follow-up: the ASD and the non-ASD (NASD) group. The following radiographic parameters were preoperatively and postoperatively measured: sagittal vertebral axis (SVA), thoracic kyphosis (TK), lumbar lordosis, pelvic tilt, and pelvic incidence (PI). Results: Eight of the 70 patients (11\%) experienced ASD after PLIF (three males and five females; age, $64.4 \pm 7.7$ years). The NASD group comprised 20 males and 42 females (age, 67.7 \pm 9.3 years). Six patients of the ASD group showed decreased L3-L4 disc height, one had L3-L4 local kyphosis, and one showed both changes. Preoperative SVA, PI, and TK were significantly smaller in the ASD group than in the NASD group $(p<0.05)$.

Conclusions: A preoperative small SVA and TK with small PI were the characteristic alignments for the risk of early-onset ASD in patients without preoperative severe sagittal spinal imbalance undergoing L4-L5 single-level PLIF.

Keywords: Sagittal spinal alignment; Posterior lumbar interbody fusion; Adjacent segment degeneration

Received Nov 1, 2017; Revised Nov 29, 2017; Accepted Dec 17, 2017

Corresponding author: Kenji Endo

Department of Orthopedic Surgery, Tokyo Medical University, 6-7-1 Nishishinjuku, Shinjuku-ku, Tokyo 160-0023, Japan

Tel: +81-3-3342-6111, Fax: +81-3-3342-5295, E-mail: kendo@tokyo-med.ac.jp 


\section{Introduction}

Lumbar spinal canal stenosis (LSCS) with segmental instability and sagittal spinal malalignment has been increasing in the elderly population [1]. LSCS unresponsive to conservative treatment is often treated using decompression surgery stabilized by instrumentation [2]. Posterior lumbar interbody fusion (PLIF) is widely performed for degenerative lumbar disorders; it provides indirect decompression of the neural foramina and allows solid fixation of unstable segments [3]. However, the segmental rigidity resulting from spinal instrumentation may increase stress at adjacent levels. The increase in rigidity of spinal segments has been reported to increase the risk of adjacent segment degeneration (ASD) after PLIF [4,5].

ASD after PLIF is usually considered a relatively longterm complication of lumbar or lumbosacral fusion $[4,5]$; the average time to diagnose radiographic lumbar instability was reported to be 25 months after transpedicular fixation [6]. Another study reported the onset of symptomatic ASD occurring at an average of 26.8 months postoperatively [7]. Age-related progressive spinal degeneration is also thought to be a major contributor to ASD [4]. The onset of ASD is reported to significantly increase in patients older than 60 years whose preoperative MRI showed disc degeneration $[8,9]$.

Another recognized risk factor for ASD is preoperative lumbo-pelvic sagittal malalignment [4,10-12], and singlelevel PLIF is now rarely performed for patients with total sagittal spinal malalignment. Single-level PLIF can only limitedly improve sagittal spinal alignment; therefore, it is generally used for patients without severe sagittal spinal imbalance. However, the risk factors for ASD after single-level intervertebral fusion are not well established. Therefore, the present study aimed to investigate the relationship between the preoperative characteristics of total spinal sagittal alignment and the early onset of ASD in patients with normal sagittal spinal alignment.

\section{Materials and Methods}

\section{Study population}

This retrospective study included 70 consecutive patients who underwent L4-L5 posterior single-segment PLIF particularly for L4-L5 lumbar degenerative disease at our hospital between January 2011 and December 2015; these patients were followed up a year later. The subjects comprised 23 males and 47 females with a mean age of $67.5 \pm 13.7$ years (range, $51-85$ years). Confounding factors were excluded by selectively recruiting patients without preoperative decreased disc height or instability at the L3-L4 single segment. Further exclusion criteria were as follows: unavailable or inadequate standing full-spine radiographs, coronal deformity $>10^{\circ}$, sagittal vertebral axis (SVA) $>100 \mathrm{~mm}$, or a previous spinal surgery or disease.

All subjects provided written informed consent after explanation of the experimental protocol. The study was approved by the Institutional Review Board of Tokyo Medical University (IRB approval no., 2165).

\section{Study procedures and radiological parameters}

Lateral standing radiographs were obtained by digital slot-scan radiography using a Sonialvision Safire fluoroscopy system (Shimadzu Corporation, Kyoto, Japan), with entrance surface doses of $0.07 \mathrm{mGy}$ and $0.21 \mathrm{mGy}$, following a standardized protocol with the patient standing in a neutral fists-on-clavicle position [13]. The following spinal parameters were assessed before surgery and at 1 year after surgery: C7-S1 SVA, T4-T12 thoracic kyphosis (TK) angle, lumbar lordosis (LL), pelvic tilt (PT), and pelvic incidence (PI) [13].

\section{Definition of early-onset radiographic adjacent seg- ment degeneration}

Radiographic evaluation was performed 1 year after the operation. Postoperative progression of radiologic ASD [14] was defined as follows: a decrease of more than $3 \mathrm{~mm}$ in disc height, an intervertebral angle at flexion less than $-5^{\circ}$, and a progress of slippage greater than $3 \mathrm{~mm}$ compared with the preoperative flexion and extension lateral radiographs. The patients were divided into two groups based on the progression of L3-L4 degeneration at final follow-up: the ASD and non-ASD (NASD) groups.

\section{Statistical analysis}

Factors associated with the development of early-onset ASD were evaluated using logistic regression analysis. The Tukey-Kramer HSD test was used to assess intergroup differences between the ASD and NASD groups to evaluate the outcome measurements of sagittal spinal 
parameters. Correlations between spinopelvic parameters were evaluated using Spearman's rank correlation coefficients. Values are expressed as means \pm standard deviation. All statistical analyses were performed using the JMP software ver. 8.0 (SAS Institute Inc., Cary, NC, USA). All $p<0.05$ were considered statistically significant.

\section{Results}

Eight of the 70 patients (11\%) exhibited L3-L4 ASD after single-level L4-L5 PLIF; these formed the ASD group (three males and five females; mean age, 64.4 \pm 7.7 years).
The remaining patients formed the NASD group, which comprised 20 males and 42 females (mean age, 67.7 \pm 9.3 years) (Table 1). Six patients from the ASD group showed decreased L3-L4 disc height, one had L3-L4 local kyphosis, and one showed both changes. None of the ASD group patients exhibited additional symptoms or underwent reoperation because of the ASD during the year after the surgery. In all the subjects, the total spinal sagittal alignment did not change significantly between the preoperative and postoperative evaluations. SVA, TK, and PI were significantly smaller in the ASD group than in the NASD group $(p<0.05)$ (Tables 2, 3). In the ASD group, the

Table 1. Morphometric characteristics of patients

\begin{tabular}{lccc} 
Characteristic & ASD group & NASD group & $p$-value \\
Age (yr) & $64.4 \pm 7.7$ & $67.7 \pm 9.3$ & 0.33 \\
Sex (male/female) & $3 / 5$ & $20 / 42$ & 0.77 \\
\hline Diagnosis & & & 53 \\
$\quad$ Degenerative spondylolisthesis & 7 & 9 & 0.97 \\
\hline Degenerative disc disease & 1 & 0.97 \\
\hline
\end{tabular}

Values are presented as mean \pm standard deviation or number.

ASD, adjacent segment degeneration.

Table 2. Preoperative parameters evaluated in the study

\begin{tabular}{llccc} 
Variable & Total & ASD group & NASD group & $p$-value \\
\hline Sagittal vertebral axis $(\mathrm{mm})$ & $35.4 \pm 30.7$ & $9.4 \pm 32.0$ & $37.7 \pm 20.2$ & 0.02 \\
\hline Thoracic kyphosis $\left(^{\circ}\right)$ & $23.7 \pm 9.7$ & $17.0 \pm 7.2$ & $25.0 \pm 8.3$ & 0.01 \\
\hline $\mathrm{LL}\left(^{\circ}\right)$ & $39.1 \pm 11.8$ & $35.8 \pm 13.1$ & $39.5 \pm 11.7$ & 0.40 \\
\hline Pelvic tilt $\left(^{\circ}\right)$ & $24.1 \pm 8.3$ & $21.3 \pm 8.9$ & $24.5 \pm 8.3$ & 0.30 \\
$\mathrm{Pl}\left({ }^{\circ}\right)$ & $55.9 \pm 10.4$ & $49.8 \pm 10.3$ & $57.4 \pm 9.7$ & 0.04 \\
$\mathrm{Pl}-\mathrm{LL}\left({ }^{\circ}\right)$ & $16.9 \pm 12.4$ & $9.9 \pm 11.3$ & $17.0 \pm 11.8$ & 0.13 \\
\hline
\end{tabular}

Values are presented as mean \pm standard deviation.

ASD, adjacent segment degeneration; LL, lumbar lordosis; PI, pelvic incidence.

Table 3. Postoperative parameters evaluated in the study

\begin{tabular}{lllll} 
Variable & Total & ASD group & NASD group & $p$-value \\
Sagittal vertebral axis $(\mathrm{mm})$ & $36.0 \pm 31.5$ & $16.0 \pm 12.4$ & $35.8 \pm 32.3$ & 0.01 \\
\hline Thoracic kyphosis $\left(^{\circ}\right)$ & $24.8 \pm 9.6$ & $16.1 \pm 7.1$ & $25.5 \pm 9.2$ & 0.01 \\
$\mathrm{LL}\left(^{\circ}\right)$ & $40.4 \pm 12.2$ & $32.5 \pm 13.8$ & $41.4 \pm 11.8$ & 0.05 \\
\hline Pelvic tilt $\left(^{\circ}\right)$ & $23.8 \pm 8.0$ & $18.9 \pm 6.8$ & $24.0 \pm 8.0$ & 0.08 \\
$\mathrm{PI}\left({ }^{\circ}\right)$ & $55.7 \pm 10.4$ & $49.8 \pm 10.3$ & $57.4 \pm 9.7$ & 0.04 \\
$\mathrm{PI}-\mathrm{LL}\left({ }^{\circ}\right)$ & $15.3 \pm 11.5$ & $17.4 \pm 13.0$ & $15.0 \pm 11.4$ & 0.59 \\
\hline
\end{tabular}

Values are presented as mean \pm standard deviation.

ASD, adjacent segment degeneration; LL, lumbar lordosis; PI, pelvic incidence. 


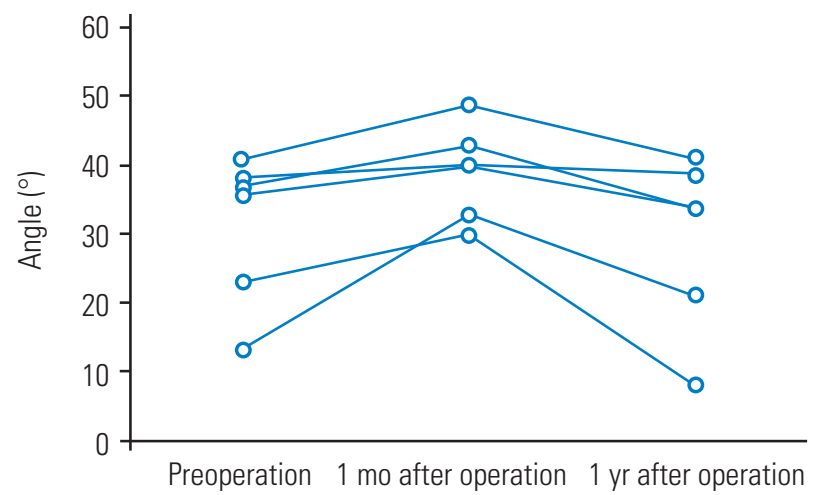

Fig. 1. Preoperative and postoperative changes in lumbar lordosis in patients with adjacent segment degeneration. Two patients were excluded because the 1-month radiograph was not available.

change in LL increased 1 month after the operation; however, at 1 year, the alignment had decreased relative to the preoperative level (Fig. 1). Postoperatively, SVA, TK, and PI were significantly smaller in the ASD group than in the NASD group $(p<0.05)$. No significant difference in PI-LL was observed between the two groups.

\section{Discussion}

Although ASD associated with spinal fusion is considered a long-term complication, this study was designed to have a follow-up of 1 year, which is a relatively short period of time, to exclude the possibility that age affects the onset of ASD after PLIF. Multiple factors are thought to contribute to ASD after PLIF. Progressive spinal degeneration with age and biomechanical change following spinal fusion are known to be major contributors to the onset of ASD [15]. Other possible risk factors for the development of ASD include laminar horizontalization and facet tropism at the level adjacent to PLIF [16] and interbody fusion from excessive distraction of the lumbar disc space [9].

It has been reported that a high PI [4] and PI-LL mismatch [10] could predispose a patient to ASD after PLIF. Kumar et al. [12] reported that the incidence of ASD was the lowest in patients with normal sagittal alignment. They reported that preoperative sagittal lumbo-pelvic malalignment produced biomechanical alteration at the adjacent level after PLIF, thereby inducing early-onset ASD. A cadaver study revealed that lumbar hypo-lordosis after posterior fusion increases the posterior shear force at the proximal adjacent segment [11]. In contrast, our results showed that the characteristic patterns in the ASD

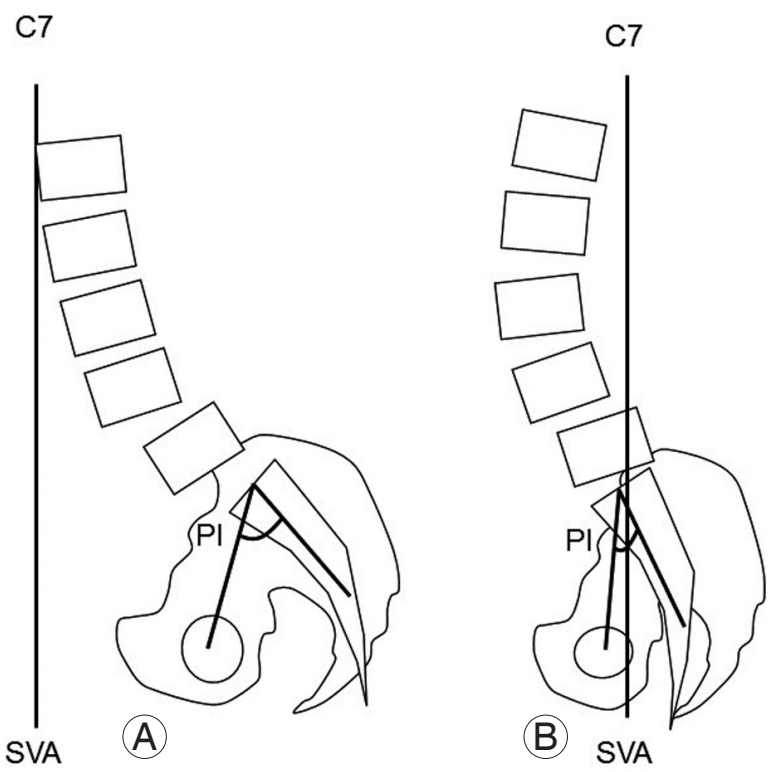

Fig. 2. Typical lumbar alignment with adjacent segment degeneration. (A) Type 1: small lumbar lordosis and large SVA with large PI. (B) Type 2: small SVA with small PI. SVA, sagittal vertebral axis; PI, pelvic incidence.

group were preoperative small SVA, PI, and TK. Reasons for these discrepancies could include our exclusion of patients with severe spinal imbalance and that our subjects were relatively well compensated by the thoracic spine and pelvis, because their total spinal sagittal alignment did not change significantly after the surgery.

Based on our results and those of previous studies $[4,10]$, the total sagittal alignment characteristics of ASD can be divided into two types: large SVA and small LL with large PI (type I) and small SVA and TK with small PI (type II) (Fig. 2). The type 1 characteristics disrupt compensation by other spinal regions, including the pelvis, whereas the type 2 characteristics maintain compensation by the thoracic spine and pelvis. This is similar to the type II classification of Roussouly and Nnadi [13], who described a flat back appearance with small PI, with the axial load increasing because of the loss of spinal curvature. PI is equal to the sum of PT and the sacral slope (SS); thus, a patient's ability to vary SS or PT to compensate for sagittal imbalance depends on the size of PI. Patients with a small PI do not have adequate capacity to be compensated by PT required to restore balance. In compensated spinal sagittal alignment, it is likely that a flat back with small PI is a key factor in promoting additional L3-L4 intervertebral mechanical load after single-level L4-L5 PLIF. Our findings showed that ASD after PLIF manifested mainly 
as a decrease in disc height, which was observed in six out of the eight ASD group patients without instability. A previous study reported that low LL and low SS were risk factors for a second or third occurrence of ASD [17]. Surgical planning should consider the evaluation of total spinal sagittal alignment, including the pelvis. Even in compensated spinal sagittal alignment, a smaller PI could be a potential risk factor for early-onset L3-L4 disc degeneration after L4-L5 PLIF.

This study had some limitations. The number of subjects was relatively small because of the strict inclusion criteria, which selected patients only with L4-L5 PLIF without additional decompression and corrective surgery, as well as exclusion of severe spinal sagittal malalignment cases. ASD is thought to be induced by multifactorial causes, and we plan to evaluate other factors and clinical outcomes in future studies.

\section{Conclusions}

The preoperative small SVA and TK with small PI were the characteristic alignments for the risk of early-onset ASD in patients without preoperative severe sagittal spinal imbalance undergoing L4-L5 single-level PLIF.

\section{Conflict of Interest}

No potential conflict of interest relevant to this article was reported.

\section{Acknowledgments}

We are indebted to the medical editors from the Department of International Medical Communications of Tokyo Medical University for editing and reviewing the English manuscript. The authors would also like to thank Ms. Yuri Amamizu of the Department of Orthopedic Surgery for assistance with preparing the initial English manuscript.

\section{References}

1. Cloyd JM, Acosta FL Jr, Ames CP. Complications and outcomes of lumbar spine surgery in elderly people: a review of the literature. J Am Geriatr Soc 2008;56:1318-27.

2. Yone K, Sakou T, Kawauchi Y, Yamaguchi M, Yanase M. Indication of fusion for lumbar spinal ste- nosis in elderly patients and its significance. Spine (Phila Pa 1976) 1996;21:242-8.

3. Starkweather A. Posterior lumbar interbody fusion: an old concept with new techniques. J Neurosci Nurs 2006;38:13-20, 30.

4. Nakashima H, Kawakami N, Tsuji T, et al. Adjacent segment disease after posterior lumbar interbody fusion: based on cases with a minimum of 10 years of follow-up. Spine (Phila Pa 1976) 2015;40:E831-41.

5. Kaito T, Hosono N, Mukai Y, Makino T, Fuji T, Yonenobu K. Induction of early degeneration of the adjacent segment after posterior lumbar interbody fusion by excessive distraction of lumbar disc space. J Neurosurg Spine 2010;12:671-9.

6. Aota Y, Kumano K, Hirabayashi S. Postfusion instability at the adjacent segments after rigid pedicle screw fixation for degenerative lumbar spinal disorders. J Spinal Disord 1995;8:464-73.

7. Etebar S, Cahill DW. Risk factors for adjacentsegment failure following lumbar fixation with rigid instrumentation for degenerative instability. J Neurosurg 1999;90(2 Suppl):163-9.

8. Ahn DK, Park HS, Choi DJ, Kim KS, Yang SJ. Survival and prognostic analysis of adjacent segments after spinal fusion. Clin Orthop Surg 2010;2:140-7.

9. Lee CS, Hwang CJ, Lee SW, et al. Risk factors for adjacent segment disease after lumbar fusion. Eur Spine J 2009;18:1637-43.

10. Rothenfluh DA, Mueller DA, Rothenfluh E, Min K. Pelvic incidence-lumbar lordosis mismatch predisposes to adjacent segment disease after lumbar spinal fusion. Eur Spine J 2015;24:1251-8.

11. Djurasovic MO, Carreon LY, Glassman SD, Dimar JR 2nd, Puno RM, Johnson JR. Sagittal alignment as a risk factor for adjacent level degeneration: a casecontrol study. Orthopedics 2008;31:546.

12. Kumar MN, Baklanov A, Chopin D. Correlation between sagittal plane changes and adjacent segment degeneration following lumbar spine fusion. Eur Spine J 2001;10:314-9.

13. Roussouly P, Nnadi C. Sagittal plane deformity: an overview of interpretation and management. Eur Spine J 2010;19:1824-36.

14. Okuda S, Iwasaki M, Miyauchi A, Aono H, Morita M, Yamamoto T. Risk factors for adjacent segment degeneration after PLIF. Spine (Phila Pa 1976) 2004;29:1535-40. 
15. Chen CS, Cheng CK, Liu CL. A biomechanical comparison of posterolateral fusion and posterior fusion in the lumbar spine. J Spinal Disord Tech 2002;15:53-63.

16. Okuda S, Oda T, Miyauchi A, et al. Lamina horizontalization and facet tropism as the risk factors for adjacent segment degeneration after PLIF. Spine (Phila Pa 1976) 2008;33:2754-8.
17. Okuda S, Oda T, Yamasaki R, Maeno T, Iwasaki M. Repeated adjacent-segment degeneration after posterior lumbar interbody fusion. J Neurosurg Spine 2014;20:538-41. 